\title{
Construction of a high-density SNP-based genetic map and identification of fruit- related QTLs and candidate genes in peach [Prunus persica (L.) Batsch]
}

Pei Shi, Ze Xu, Shaoyu Zhang, Xianju Wang, Xiaofei Ma, Jicheng Zheng, Libo Xing, Dong Zhang, Juanjuan Ma, Mingyu Han and Caiping Zhao ${ }^{*}$ (1)

\begin{abstract}
Background: High-density genetic mapping is a valuable tool for mapping loci that control specific traits for perennial fruit trees. Peach is an economically important fruit tree and a model Rosaceae species for genomic and genetic research. In peach, even though many molecular markers, genetic maps and QTL mappings have been reported, further research on the improvement of marker numbers, map densities, QTL accuracy and candidate gene identification is still warranted.

Results: A high-density single nucleotide polymorphism (SNP)-based peach linkage map was constructed using specific locus amplified fragment sequencing (SLAF-seq). This genetic map consisted of 7998 SLAF markers, spanning $1098.79 \mathrm{cM}$ with an average distance of $0.17 \mathrm{cM}$ between adjacent markers. A total of 40 QTLs and 885 annotated candidate genes were detected for 10 fruit-related traits, including fruit weight (FW), fruit diameter (FD), percentage of red skin colour (PSC), eating quality (EQ), fruit flavour (FV), red in flesh (RF), red around pit (RP), adherence to pit (AP), fruit development period (FDP) and fruit fibre content (FFC). Eighteen QTLs for soluble solid content (SSC) were identified along LGs 1, 4, 5, and 6 in 2015 and 2016, and 540 genes were annotated in QTL intervals. Thirty-two QTLs for fruit acidity content (FA) were detected on LG1, and 2, 4, 5, 6, and 1232 candidate genes were identified. The expression profiles of 2 candidate genes for SSC and 4 for FA were analysed in parents and their offspring.

Conclusions: We constructed a high-density genetic map in peach based on SLAF-seq, which may contribute to the identification of important agronomic trait loci. Ninety QTLs for 12 fruit-related traits were identified, most of which overlapped with previous reports, and some new QTLs were obtained. A large number of candidate genes for fruitrelated traits were screened and identified. These results may improve our understanding of the genetic control of fruit quality traits and provide useful information in marker-assisted selection for fruit quality in peach breeding programmes.
\end{abstract}

Keywords: SNP-based map, Peach, SLAF, QTL, Fruit quality-related traits

\footnotetext{
* Correspondence: zhcc@nwsuaf.edu.cn

College of horticulture, Northwest A\&F University, Yangling 712100, Shaanxi, China
} 


\section{Background}

Peach [Prunus persica (L.) Batsch] is well-known as a delicious and healthy summer fruit in the temperate regions of the world with a total production of approximately 2.47 million tonnes worldwide in 2017 (http://www.fao.org/faostat/en/\#data). Peach is an important perennial fruit tree species with few chromosomes $(2 \mathrm{n}=2 \mathrm{x}=16)$, a relatively small genome $(\sim 230$ $\mathrm{Mbp}$ ), and a short juvenile period (2-4 years) [1]. These traits make peach a model plant in Rosaceae fruit trees for genetic map construction, important agronomic trait location, and target candidate gene identification.

Fruit quality is a complex trait involving fruit appearance, texture, taste, flavour and so on. Infante et al. (2008) overviewed the fruit quality evaluation through physical, chemical and sensorial parameters, and the inheritance and molecular breeding of the main fruit quality traits in peach [2]. Most of these quality traits are quantitatively inherited and controlled by multiple loci. The construction of genetic maps and quantitative trait loci (QTL) analysis are effective strategies for the identification of candidate genes associated with fruit quality traits [3].

The first peach map was reported by Chaparro et al. using intraspecific F2 progeny, including 83 random amplified polymorphic DNA (RAPD) markers, 1 isozyme and 4 morphological markers [4]. Subsequently, with the development of molecular marker technology, numerous peach genetic linkage maps were constructed [5-9].

Sosinski et al. detected 12 QTLs for soluble solids, pH, cold tolerance, maturation date, and fruit size [5]. Dirlewanger et al. identified 32 QTLs related to sugar and acid contents, and epistasis was observed between QTLs [10]. The molecular markers tightly linked to several Mendelian agronomic characteristics were also detected. Freestone (F) was located in G4, low acid (D) and peach (G) in G5, and pollen sterility (Ps), flat (S), and aborting fruit (Af) in G6 [6]. A cluster of QTLs for fruit weight (FW), juice total soluble solids (SSC), and juice titratable acidity (FA) was found at a distal position on G4 close to the endoPG CAPS marker, and QTLs for maturity date (MD) were also found on G4 [7]. Fresnedo-Ramírez et al. identified 5 QTLs that accounted for up to $~ 29 \%$ of the phenotypic variation of fruit equatorial diameter (FD) and up to $\sim 17 \%$ of fresh weight (FW) [11]. Hernández Mora et al. identified 47 QTLs for the seven most important agronomic traits of peach by the integrated analysis of 18 families from different European breeding programmes [12].

Significant QTL effects were detected on linkage group 4 for fruit mealiness (M) and flesh bleeding (FBL) and on linkage group 5 for flesh browning (FBr) [8]. Sánchez et al. identified QTLs for volatile compounds in peach fruit, and QTL mapping showed clustering of volatile QTLs included in the same volatile modules. A unique locus at the top of LG4 controlled the monoterpene module [13]. Eduardo et al. identified three major QTLs for nonanal, linalool, and p-menth-1-en-9-al in linkage group 4. The genes encoding two putative terpene synthases and one lipoxygenase (Lox) might be involved in the biosynthesis of linalool and p-menth-1-en-9-al and nonanal, respectively [14]. Bielenberg et al. detected ten QTLs for chilling requirement (CR) and nineteen QTLs for bloom date (BD) [15]. Two stony hard (SH) phenotype-related QTLs were found in linkage group LG6. Three genes (Prupe.6G150900.1, Prupe.6G147600.1 and Prupe.6G156500.1) were identified as candidates for the $\mathrm{SH}$ trait [9].

In recent years, expression QTLs (eQTLs) was used to identify the candidate genes located inside QTL regions for the fruit quality traits. For example, García-Gómez et al. (2019) identified QTLs linked to fruit quality traits of apricot, and obtained a candidate gene, MYB transcription factor, for skin colour in LG3, and three candidate genes for the SSC in LG4 through gene expression analysis [16]. Carrasco-Valenzuela et al. (2019) had developed an integrative analysis involving conventional QTLs, eQTLs (expression QTLs), and transcriptome profiling to identify candidate genes involved in peach fruit softening rate, and indicated that auxin biosynthetic related genes triggered fast softening in melting peach fruit [17]. Although many molecular marker, genetic map and QTL analyses have been reported, the accuracy of QTL mapping needs to be improved, and concise information on the number and position of the genes determining the inheritance of a given trait is lacking [12].

In this study, we constructed a high-density genetic map using SNP markers which be developed through specific-locus amplified fragment sequencing (SLAFseq), and then we identified stable QTLs associated with 12 fruit quality traits (including fruit weight, fruit diameter, fruit skin colour, red in flesh, red around pit, adherence to pit, fruit development period, fruit fibre content, fruit flavor, eating quality, soluble solid content and fruit acidity content) for two years in an F1 population. Subsequently, we screened the candidate genes located inside these stable QTL regions and verified them through gene expression analysis using qPCR. Our results will be beneficial for understanding the genetic basis for the formation of peach fruit quality, thus providing a theoretical basis for improving fruit quality via MAS and / or genetic manipulation.

\section{Results}

Fruit-related trait phenotypic identification

Twelve fruit-related traits from 202 individuals of the F1 population were analysed in 2015 and 2016. The group of quantitative traits included fruit development period (FDP), fruit weight (FW), fruit diameter (FD), percentage 
of red skin colour (PSC), eating quality (EQ), fruit flavour (FV), soluble solid content (SSC), and fruit acidity content (FA). FW, FD, PSC, FV and EQ had normal distributions in progeny, and FDP showed a significant non-normal distribution (Fig. 1). Therefore, FDP was treated as a qualitative trait in the subsequent analysis. SSC and FA of parents and $F_{1}$ progenies were measured during fruit storage. SSC showed a slight upward trend during storage in both 2015 and 2016, while FA increased in 2016 and remained stable in 2015. In addition, the SSC and FA in 2016 were higher than those in 2015 (Fig. 2). The group of qualitative traits included red in flesh (RF), red around pit (RP), adherence to pit (AP), fruit fibre content (FFC) and FDP. QTLs for these qualitative traits were detected using the KruskalWallis test.

High-density genetic linkage map construction for peach A total of 7998 out of 8037 high-quality polymorphic SLAF markers were distributed into eight linkage groups (LGs) according to their locations in the Prunus persica genome (Fig. S1). The integrated genetic map spanned $1098.79 \mathrm{cM}$, with an average distance of $0.17 \mathrm{cM}$ between the adjacent markers. LG1 was the largest LG containing 1699 markers, covering $143.89 \mathrm{cM}$, with an average distance of only $0.08 \mathrm{cM}$ and a maximum gap of only 2.67 cM being observed between the adjacent markers. LG8 had the fewest markers of 386 and spanned a length of $113.03 \mathrm{cM}$, with an average distance of $0.29 \mathrm{cM}$ and a maximum gap of $5.86 \mathrm{cM}$ being observed between the adjacent markers (Table 1). In addition, the collinearity of mapping markers between the physical and genetic maps was determined with Spearman correlations. A high level of collinearity was found, represented by scores of less than or equal to 1.0 (Fig. S2).

\section{QTLs identified for fruit-related traits}

The QTL analysis of 12 fruit-related traits was performed by using MapQTL6.0 software with the above SLAF-based high density linkage map. For FW, FD, PSC, EQ and FV with normal distributions, a total of 21 QTLs were detected. Four QTLs were identified for FW, of which two were located on LG 4 (7 and 6.4\%), the third was located on LG 5 (5.3\%), and the fourth was located on LG 6 (5.8\%). Four QTLs were identified for FD, two of which were located on LG 4 (5.9 and 10.3\%), and the other two were located on LG 5 (6.8 and 6.0\%). Five QTLs were identified for PSC, two of which were located on LG 4 (7 and 7.5\%), and the remaining 3 QTLs were located on LG 1 (6.4\%), LG 3 (4.9\%), and LG 6 (6.6\%). Two QTLs for EQ were located on LG 1 (7 and 6.8\%). Six QTLs were identified for FV, of which two were located on LG 1 (7.7 and 8.1\%), two were located on LG5 (13.9 and 5.3\%), and two were located on LG3 (10.4\%) and LG4 (6.7\%) (Table 2).

In total, 19 QTLs were identified for 5 qualitative traits, RF, RP, AP, FDP and FFC (the same QTL in 2 years was considered as one QTL). Two overlapping QTLs for RF were located on LG 1. Four QTLs were identified for RP on LG 1 in both 2015 and 2016. Five QTLs were identified for AP, three of which were located on LG 4, and the other two were located on LG 3 and LG 7. Four QTLs were identified for FDP, of which one was located on LG 7, and the other three were located on LG 4. Four QTLs were identified for FFC, of which two were located on LG 3, and the other two were located on LG 2 and LG 7 (Table 2, Fig. S3). Furthermore, most QTLs were confirmed in both 2015 and 2016 and in the same location (Table 2, Fig. S3). The stability of loci for these qualitative traits in different years confirmed the reliability of QTLs.

Dynamic QTLs for SSC and FA based on measured data from the peach fruit storage period were detected in 2015 and 2016. A total of 18 QTLs for SSC were identified along LGs 1, 4, 5, and 6 in 2015 and 2016. Ten QTLs on LG1 were located at $41.102 \sim 42.728 \mathrm{cM}$ (Pp01:13,177,641.. $\quad 15,291,518), \quad 66.897 \sim 67.265 \mathrm{cM}$ (Pp01: $\quad 24,241,572 . . \quad 24,284,070), \quad 75.744 \sim 77.560 \mathrm{cM}$ (Pp01: 25,898,412.. 26,757,867), $91.990 \sim 120.785 \mathrm{cM}$ (Pp01: 29,246,116.. 42,673,647). Two QTLs on LG4 were located at 149.153 149.934 cM (Pp04: 28,137,742.. 30, 186,866). Two QTLs on LG5 were located at 27.451 30.996 cM (Pp05: 846,656.. 1,094,221). Four QTLs on LG6 were located at $29.965 \sim 37.885 \mathrm{cM}$ (Pp06: 4,716, 586.. 5,153,723) (Fig. 3, Fig. S3). Thirty-two QTLs for FA were detected: 3 QTLs were located on LG1, 3 QTLs were on LG2, 9 QTLs were on LG4, 6 QTLs were on LG5, and 11 QTLs were on LG6 (Fig. 3, Fig. S3).

\section{Potential candidate genes in the QTL interval of fruit- related traits}

Potential candidate genes involved in 12 fruit-related traits were investigated according to their QTL intervals with the physical positions on the peach genome. For SSC, 540 annotated candidate genes were identified in the above 18 QTL regions (Table S1). These candidate genes are involved in such processes as the biosynthesis of secondary metabolites, fructose and mannose metabolism, and fatty acid metabolism (Fig. S4).

For FA, 1232 annotated genes were detected in 32 QTL regions (Table S1). These candidate genes are focused on such processes as the biosynthesis of secondary metabolites, protein processing in the endoplasmic reticulum, starch and sucrose metabolism, carbon fixation in photosynthetic organisms, and C5-branched dibasic acid metabolism (Fig. S5). These candidate genes also included such genes as ATP-citrate lyase, the 


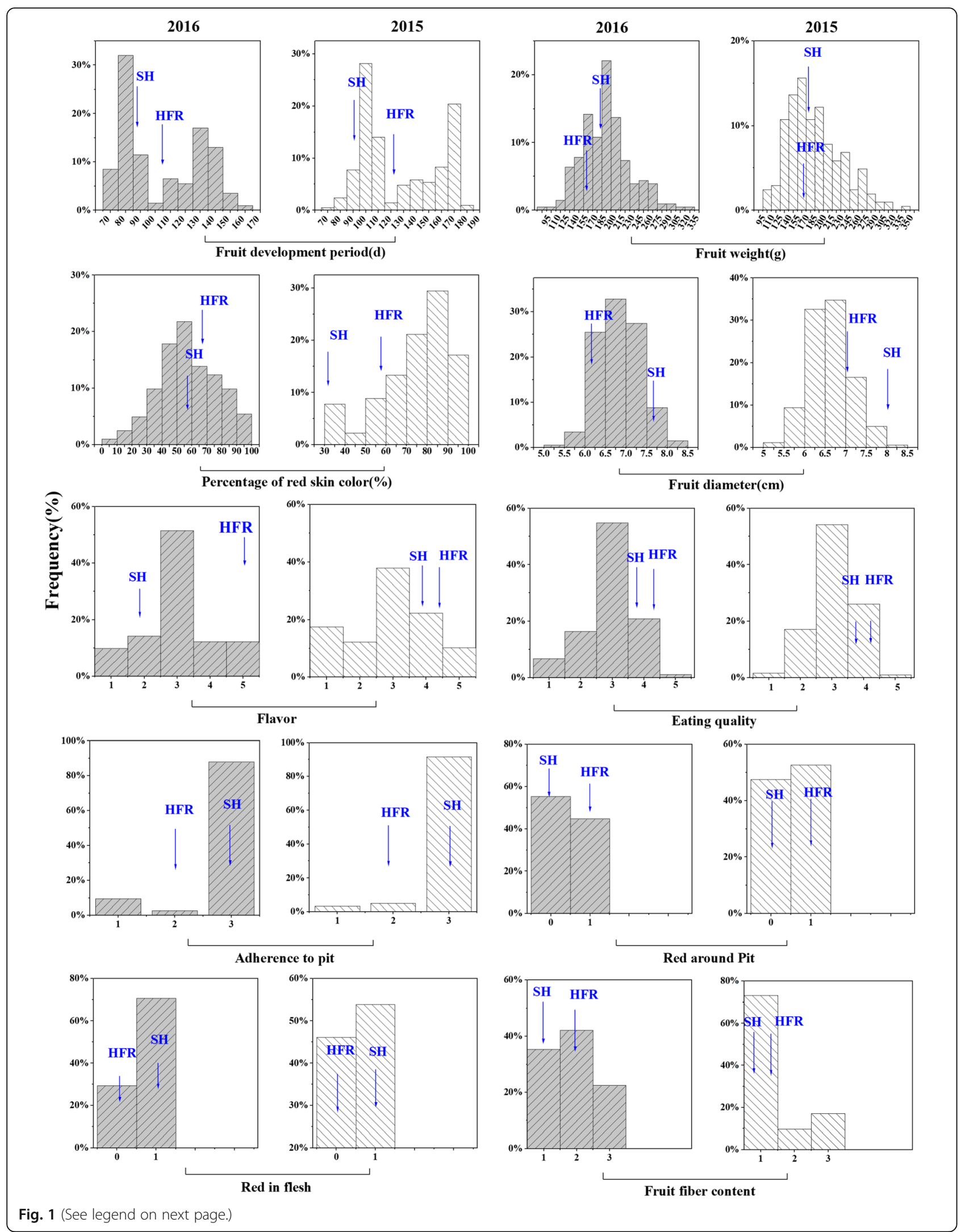


(See figure on previous page.)

Fig. 1 Distribution of phenotypes for fruit quality traits (including fruit development period, fruit weight, fruit diameter, percentage of red skin colour, red in flesh, red around pit, adherence to pit, eating quality), fruit flavour and fruit fibre content measured in 2015 and 2016 on the F1 progeny. Notes: Fruit flavour ( $1=$ sour, $2=$ sour-sweet, $3=$ water sweet, $4=$ sweet, $5=$ rich sweet $)$; eating quality $(1=$ extremely poor, $2=$ poor, $3=$ fair, 4 = good, 5 = excellent); adherence to pit $(1=$ freestone, $2=$ semi-freestone, $3=$ clingstone); red around pit $(0=$ no red, $1=$ red); fruit fibre content $(1=$ few, 2 = intermediate, $3=$ many); red in flesh $(0=$ no red overlay, $1=$ red overlay). The values of the parents 'Shahong' $(\mathrm{SH})$ and

'Hongfurong' (HFR) are indicated by arrows

aluminium-activated malate transporter, vacuolar proton ATPase, and the auxin efflux carrier family protein, which may be related to FA. In addition, sucrose transporter 2, UDP-glucose 6-dehydrogenase family protein, 6-phosphogluconolactonase, fructokinase-like 2, sugar transporter protein, fructose-2,6-bisphosphatase and glucose-6-phosphate dehydrogenase, which may be related to the sugar content of the fruit, have been identified for FA (Table S1).

For the remaining 10 fruit-related traits, 885 annotated candidate genes on QTL regions were detected, 130 for FW and FD (FW and FD candidate genes completely overlap), 11 for PSC, 21 for EQ, 186 for RF, 125 for AP, 3 for RP, 6 for FDP, 401 for FV and 2 for FFC (Table S2). Candidate genes for these fruit quality traits were significantly enriched in the secondary metabolic pathway (Fig. S6-S9). In addition, candidate genes for FW are also involved in the biosynthesis of amino acids and protein processing in the endoplasmic reticulum (Fig. S6). Candidate genes for FV are focused on such processes as carbon metabolism, citrate cycle (TCA cycle), and fructose and mannose metabolism (Fig. S7). Candidate genes for EQ are also enriched in such processes as starch and sucrose metabolism (Fig. S8), and RFs are enriched in, for instance, $A B C$ transporters and phenylpropanoid biosynthesis (Fig. S9).

\section{Expression analysis of selected candidate genes for SSC and FA}

To further confirm the relationship between the candidate genes and fruit SSC and FA, we selected 6 candidate genes, 2 for SSC and 4 for FA, to evaluate their expression in parents and representative offspring. For the SSC trait, HFR, 24-5, 24-16 and 25-15 showed high SSC, while SH, 22-10, 22-11, and 25-37 had low SSC during fruit storage (Fig. 4). qRT-PCR results showed that Prupe.1G250100 and Prupe.6G076900 exhibited significant differences between SH and HFR at the mature stage. The expression levels of Prupe.1G250100 and Prupe.6G076900 in the 6 hybrids were negatively correlated
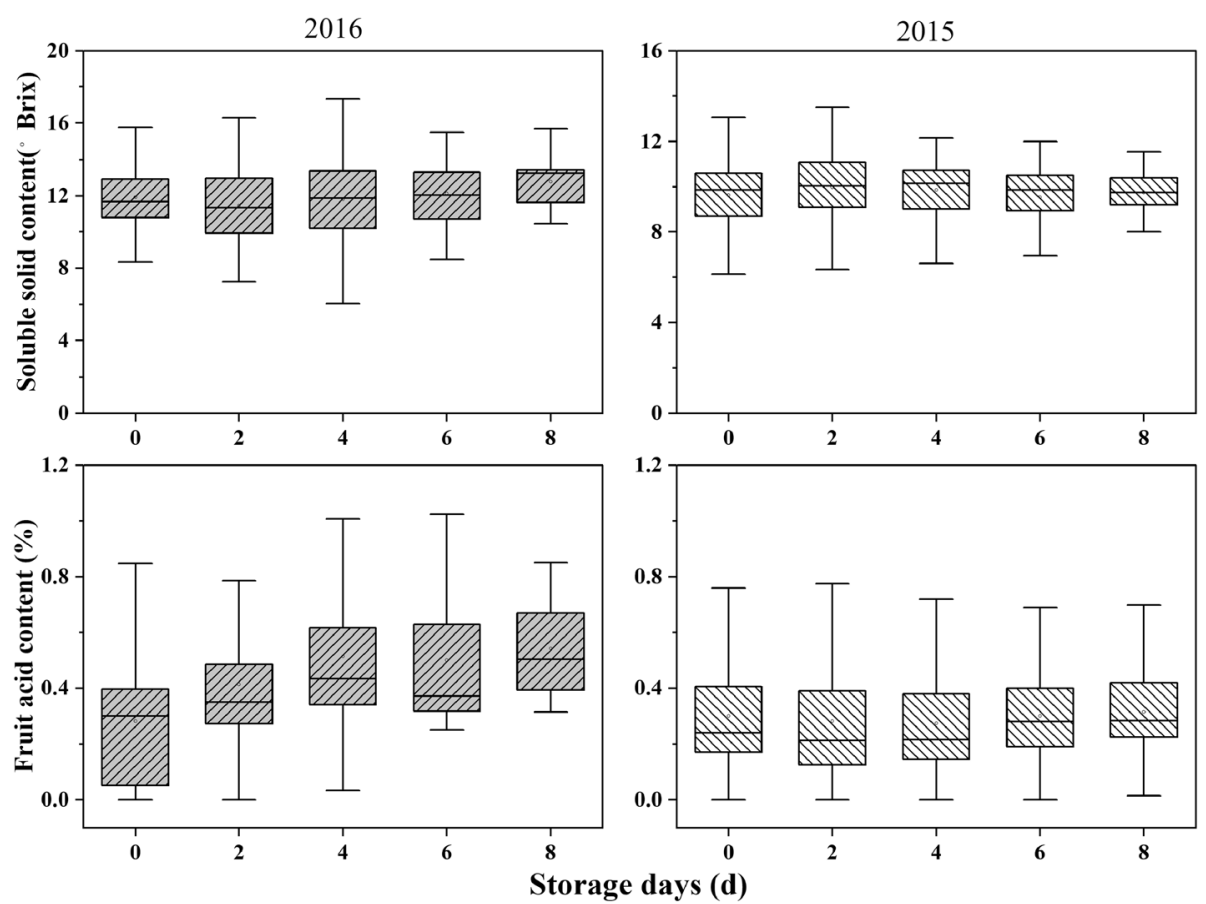

Fig. 2 Boxplot distributions of soluble solid content and fruit acidity content in 'Shahong' $\times$ 'Hongfurong' F1 progeny measured during storage at $25^{\circ} \mathrm{C}$ for the years 2015 and 2016 
Table 1 Distribution of mapped markers among eight linkage groups in peach

\begin{tabular}{lllll}
\hline Linkage Group ID & Total Marker & Total Distance (cM) & Average Distance (cM) & Max Gap \\
\hline Chr1 & 1699 & 143.89 & 0.08 & 2.67 \\
Chr2 & 1397 & 107.65 & 0.08 & 4.51 \\
Chr3 & 1025 & 184.81 & 0.18 & 7.12 \\
Chr4 & 1462 & 167.07 & 0.11 & 11.70 \\
Chr5 & 506 & 148.45 & 0.29 & 15.45 \\
Chr6 & 740 & 116.71 & 0.16 & 5.30 \\
Chr7 & 783 & 117.17 & 0.15 & 5.44 \\
Chr8 & 386 & 113.03 & 0.29 & 5.86 \\
Total & 7998 & 1098.79 & 0.17 & 15.45 \\
\hline
\end{tabular}

with changes in SSC (Fig. 5). Prupe.1G250100 and Prupe.6G076900 encode ATP-citrate lyase A-2 and Oglycosyl hydrolase family 17 proteins, respectively.

For the FA trait, $\mathrm{SH}, 23-6,24-25$ and $25-8$ showed high FA, while HFR, 25-14, 25-38 and 23-8 had low FA during fruit storage (Fig. 4). The expression of Prupe.1G289200 in SH and offspring with high FA was higher than that in HFR and offspring with low FA (Fig. 5). The expression of Prupe.4G225100, Prupe.1G284300 and Prupe.6G307600 was more highly expressed in low FA fruits than in high FA fruits at the mature stage (Fig. 5). Prupe.1G289200, Prupe.1G284300, Prupe.4G225100 and Prupe.6G307600 encode 6-phosphogluconolactonase, UDPglucose 6-dehydrogenase, ATPase $\mathrm{H}+$ transporting $\mathrm{V} 0$ subunit E (ATPase, V0 complex, subunit E) and glucose6-phosphate dehydrogenase, respectively. Because Prupe.1G284300 and Prupe.6G307600 are involved in the sugar metabolic pathway, we also detected the expression of Prupe.1G284300 and Prupe.6G307600 in parents and offspring with different SSCs. The results showed that the expression of Prupe.1G284300 was significantly positively correlated with the fruit SSC (Fig. 5).

\section{Discussion}

\section{High-density SNP genetic map for peach}

Molecular markers, such as AFLPs, RAPDs, RFLPs, SSRs and SNPs, have been widely used for genetic map construction and QTL identification in peach $[4,5,14,18]$. Previously constructed peach linkage maps mainly used AFLPs, RAPDs, RFLPs and SSRs markers; due to the lower availability of these markers, the map density was not high enough and the adjacent marker gaps were larger $[4,5,18-21]$. Recently, a large number of SNP markers were used to construct genetic maps with highdensity, and to screen the QTLs of fruit quality related traits $[8,9,14,15]$. SNP is the most common genetic variation in the plant genome, and it is a highly important genetic marker for constructing a high-density genetic map and completing molecular marker-assisted breeding [22, 23]. Next-generation high-throughput sequencing technologies have recently facilitated the large-scale discovery of genome-wide SNP markers. SLAF-seq is a cost-effective technology for SNP discovery and genotyping and has been applied for genetic map construction and QTL detection in many plant species [24-29]. In addition, the high-density SNP array also was used in high-throughput genotyping, genetic map construction and genome-wide association study of fruit traits [30]. The IPSC $9 \mathrm{~K} \mathrm{SNP}$ array was commercially available for peach [31]. However, this array was more expensive, and the SNPs on the array were not evenly spaced across the peach genome with adjacent marker gaps up to $1254 \mathrm{~kb}$ for some genomic regions [31].

In this study, we constructed a high-density genetic linkage map for peach with 8 linkage groups and 7998 SLAF markers. This genetic map spanned $1098.79 \mathrm{cM}$ with an average distance of $0.17 \mathrm{cM} /$ marker. Dirlewanger et al. (1998) established a peach map covering $712 \mathrm{cM}$, and the average density between pairs of markers was $4.5 \mathrm{cM}$ [32]. Blenda et al. (2007) published a map including 151 AFLP and 21 SSR markers covering the peach genome of $737 \mathrm{cM}$ with an average marker spacing of $4.7 \mathrm{cM}$ [33]. The Pop-DG intraspecific peach linkage map covered $818.2 \mathrm{cM}$ of the peach genome with an average interval of $4.0 \mathrm{cM}$ between markers [34]. Martínez-García et al. (2013) constructed a peach map with 588 SNP markers and map coverage of $454 \mathrm{cM}$ and an average distance of $0.81 \mathrm{cM} /$ marker site [8]. Nuñez-Lillo et al. (2015) built a linkage map with 1830 SNPs and 7 SSR markers spanning $389.2 \mathrm{cM}$ distributed over eight linkage groups with an average interval of $0.21 \mathrm{cM} /$ marker pair [3]. Guo et al. (2018) published a linkage map with 1310 SNP markers spanning $454.2 \mathrm{cM}$ with an average marker distance of $0.347 \mathrm{cM}$ [9]. Compared with the published linkage maps, the genetic group constructed in our study covered a longer genetic distance and contained a higher marker density. The differences 
Table 2 Identified QTLs of 10 fruit-related traits

\begin{tabular}{|c|c|c|c|c|c|c|c|c|c|c|c|}
\hline \multicolumn{12}{|c|}{ I. QTLs of quantitative traits using interval mapping and multiple QTL model method } \\
\hline \multirow[t]{2}{*}{ Trait } & \multirow[t]{2}{*}{ Year } & \multirow[t]{2}{*}{ LG } & \multirow[t]{2}{*}{ Cofactor Marker } & \multirow[t]{2}{*}{ QTL } & \multicolumn{3}{|c|}{ Genetic Position } & \multicolumn{2}{|c|}{ Physical Position } & \multirow[t]{2}{*}{ LOD } & \multirow{2}{*}{$\begin{array}{l}\text { explained } \\
\text { variations (\%) }\end{array}$} \\
\hline & & & & & $\begin{array}{l}\text { Start } \\
(\mathrm{cM})\end{array}$ & $\begin{array}{l}\text { Final } \\
\text { (cM) }\end{array}$ & $\begin{array}{l}\text { Interval } \\
\text { (cM) }\end{array}$ & Start (bp) & Final (bp) & & \\
\hline \multirow[t]{4}{*}{ Fruit weight (FW) } & 2015 & 4 & Marker415082371 & qP-FW4.1 $1^{1}$ & 97.3 & 98.289 & 0.99 & $15,082,371$ & $16,710,388$ & 2.81 & 7.0 \\
\hline & 2016 & 4 & Marker46320002 & qP-FW4.2 $2^{2}$ & 45.027 & 45.421 & 0.39 & $6,316,366$ & $6,320,223$ & 2.47 & 6.4 \\
\hline & 2016 & 5 & Marker57632555 & qP-FW5.12 & 55.908 & 56.194 & 0.29 & $7,318,892$ & $7,816,084$ & 2.2 & 5.3 \\
\hline & 2016 & 6 & Marker628159465 & qP-FW6. $1^{2}$ & 108.055 & 108.055 & 0.00 & $28,159,465$ & $28,159,465$ & 2.54 & 5.8 \\
\hline \multirow[t]{4}{*}{ Fruit diameter (FD) } & 2015 & 4 & Marker415082371 & qP-FD4.1 ${ }^{1}$ & 97.3 & 98.289 & 0.99 & $15,082,371$ & $16,710,388$ & 3.84 & 10.3 \\
\hline & 2016 & 4 & Marker46320002 & qP-FD $4.2^{2}$ & 45.027 & 45.027 & 0.00 & $6,320,002$ & $6,320,002$ & 2.59 & 5.9 \\
\hline & 2015 & 5 & Marker57664342 & qP-FD5. $1^{1}$ & 55.908 & 55.908 & 0.00 & $7,664,342$ & $7,664,342$ & 2.16 & 6.8 \\
\hline & 2016 & 5 & Marker57632555 & qP-FD5.2 $2^{2}$ & 55.908 & 56.194 & 0.29 & $7,318,892$ & $7,816,084$ & 2.67 & 6.0 \\
\hline \multirow{5}{*}{$\begin{array}{l}\text { Percentage of red skin } \\
\text { color (PSC) }\end{array}$} & 2016 & 1 & Marker121499106 & QP-PSC1.12 & 56.814 & 57.179 & 0.37 & $21,487,305$ & $21,513,105$ & 2.8 & 6.4 \\
\hline & 2016 & 3 & Marker310648307 & qP-PSC $3.1^{1}$ & 108.701 & 108.709 & 0.08 & $10,648,307$ & $10,838,614$ & 2.06 & 4.9 \\
\hline & 2015 & 4 & Marker419804890 & qP-PSC $4.1^{1}$ & 121.898 & 121.915 & 0.02 & $19,804,890$ & $19,842,357$ & 2.58 & 7.0 \\
\hline & 2016 & 4 & Marker428152553 & qP-PSC $4.2^{2}$ & 149.991 & 150.074 & 0.08 & $28,152,553$ & $28,561,890$ & 3.27 & 7.5 \\
\hline & 2015 & 6 & Marker626821847 & qP-PSC6. $1^{1}$ & 99.526 & 99.537 & 0.01 & $26,821,847$ & $26,822,849$ & 2.52 & 6.6 \\
\hline \multirow[t]{2}{*}{ Eating quality (EQ) } & 2016 & 1 & Marker121482495 & QP-EQ $1.1^{2}$ & 55.794 & 56.344 & 0.55 & $21,332,095$ & $21,482,979$ & 2.55 & 7.0 \\
\hline & 2015 & 1 & Marker126704889 & QP-EQ $1.2^{1}$ & 77.286 & 77.458 & 0.17 & $26,561,825$ & $26,750,141$ & 2.56 & 6.8 \\
\hline \multirow[t]{6}{*}{ Flavor (FV) } & 2016 & 1 & Marker127158729 & qP-FV1.1 ${ }^{2}$ & 78.031 & 80.198 & 2.17 & $26,694,116$ & $27,334,450$ & 3.36 & 7.7 \\
\hline & 2016 & 1 & Marker128065739 & qP-FV1.2 $2^{2}$ & 83.271 & 86.952 & 3.68 & $27,653,862$ & $28,166,262$ & 3.52 & 8.1 \\
\hline & 2016 & 3 & Marker33722468 & qP-FV $3.1^{2}$ & 31.417 & 70.374 & 38.96 & $3,128,322$ & $4,476,565$ & 4.56 & 10.4 \\
\hline & 2015 & 4 & Marker417553353 & qP-FV4. $1^{1}$ & 100.574 & 103.631 & 3.06 & $15,270,537$ & $17,635,472$ & 2.99 & 6.7 \\
\hline & 2015 & 5 & Marker5638231 & qP-FV5. $1^{1}$ & 20.72 & 20.72 & 0.00 & 638,231 & 638,231 & 2.2 & 13.9 \\
\hline & 2015 & 5 & Marker51499828 & qP-FV5.2 & 42.642 & 42.897 & 0.25 & $1,499,828$ & 1499,831 & 2.33 & 5.3 \\
\hline
\end{tabular}

II. QTLs of qualitative traits using Kruskal-Wallis method

Trai

Red in flesh (RF)

Red around pit (RP)
Year LG Cofactor marker QTL

Genetic Position

Start Final Interval (cM) (cM) (cM)

20161 Marker124532043 qP-RF1.12 $68.951 \quad 72.768 \quad 3.82$

20151 Marker124908662 qP-RF1.2. ${ }^{1}$ $\begin{array}{lll}68.826 & 76.049 & 7.22\end{array}$ 20161 Marker126704889 qP-RP1.1 ${ }^{2}$ 20151 Marker126704889 qP-RP1.2 ${ }^{1}$

20161 Marker126747840 qP-RP1.32

20151 Marker126747840 qP-RP1.4 ${ }^{1}$

20161 Marker126757867 qP-RP1.5²

20151 Marker126757867 qP-RP1.6 ${ }^{1}$

20161 Marker127176678 qP-RP1.7 ${ }^{2}$

20151 Marker127176678 qP-RP1.8

20163 Marker37112788 qP-AP3.1 ${ }^{2}$

20153 Marker37112788 qP-AP3.2 ${ }^{1}$

20167 Marker715748214 qP-AP7.1 ${ }^{2}$

20157 Marker715748214 qP-AP7.2 ${ }^{1}$

20154 Marker423822119 qP-AP4.1 ${ }^{1}$

20164 Marker414000094 qP-AP4.2 ${ }^{2}$

20164 Marker418581993 gP-AP4.3 $^{2} \quad 110.133 \quad 110.133 \quad 0.00$
Physical Position $\quad K^{*} \quad$ Signif.

Start (bp) Final (bp)

$24,430,498 \quad 25,072,844 \quad 11.255 \quad * * * *$ $24,397,147 \quad 26,479,742 \quad 8.896 \quad * * * *$ $26,671,055 \quad 26,704,889 \quad 7.21 \quad * *$ $26,704,889 \quad 26,704,889 \quad 6.673 \quad * *$ $26,747,840 \quad 26,750,141 \quad 8.585 \quad * *$ $26,747,840 \quad 26,750,141 \quad 6.053 \quad * *$ 
Table 2 Identified QTLs of 10 fruit-related traits (Continued)

\begin{tabular}{|c|c|c|c|c|c|c|c|c|c|c|c|}
\hline \multirow{5}{*}{$\begin{array}{l}\text { Fruit development period } \\
\text { (FDP) }\end{array}$} & 2016 & 7 & Marker72036314 & qP-FDP7. $1^{2}$ & 2.454 & 2.521 & 0.07 & $1,838,911$ & $2,036,314$ & 6.564 & ** \\
\hline & 2015 & 7 & Marker72036314 & qP-FDP7. $2^{1}$ & 2.454 & 2.521 & 0.07 & $1,838,911$ & $2,036,314$ & 5.472 & $* *$ \\
\hline & 2016 & 4 & Marker420753150 & qP-FDP4. $1^{2}$ & 126.847 & 126.847 & 0.00 & $20,753,150$ & $20,753,150$ & 11.007 & $* * * *$ \\
\hline & 2015 & 4 & Marker45665856 & qP-FDP4.2 $2^{1}$ & 40.716 & 40.716 & 0.00 & $5,665,842$ & $5,665,856$ & 6.152 & $* *$ \\
\hline & 2015 & 4 & Marker416420904 & qP-FDP4. $3^{1}$ & 108.949 & 108.949 & 0.00 & $16,420,904$ & $16,420,904$ & 8.099 & $* * * *$ \\
\hline \multirow[t]{5}{*}{ Fruit fiber content (FFC) } & 2015 & 2 & Marker27416433 & qP-FFC2. $1^{1}$ & 30.062 & 30.353 & 0.29 & $7,416,433$ & $7,501,740$ & 4.244 & $* * * *$ \\
\hline & 2016 & 3 & Marker314273878 & qP-FFC3.1 $1^{2}$ & 124.161 & 124.346 & 0.19 & $14,273,878$ & $14,298,880$ & 3.95 & $* *$ \\
\hline & 2015 & 3 & Marker316663661 & qP-FFC3.2 ${ }^{1}$ & 138 & 138.039 & 0.04 & $16,663,659$ & $16,663,661$ & 10.159 & $* * *$ \\
\hline & 2016 & 7 & Marker76260019 & qP-FFC7.1 $1^{2}$ & 12.237 & 12.237 & 0.00 & $6,260,019$ & $6,260,019$ & 6.52 & $* *$ \\
\hline & 2015 & 7 & Marker76260019 & qP-FFC7. $2^{1}$ & 12.237 & 12.237 & 0.00 & $6,260,019$ & $6,260,019$ & 8.318 & $* *$ \\
\hline
\end{tabular}

$\mathrm{K}^{*}$ : the Kruskal-Wallis test statistic. ${ }^{* *}: 0.01 ;{ }^{* * *}: 0.001 ;{ }^{* * * *} 0.0001$

in the length and density of genetic maps may be related to the genetic distance between the parents, the number of markers, and the population size used in different studies. The increased marker number and density could enhance the mapped QTL number as well as the precision $[35,36]$. Our experiment results showed that SLAFbased SNP markers were highly effective for constructing high-density genetic maps.

\section{QTLs identified for fruit-related traits in peach}

Fruit quality is a composite trait, and breeding for fruit quality traits is complex due to the polygenic nature of the genetic control of these traits [37]. Mapping QTLs controlling fruit quality in peach has been widely reported [7, 8, 38-41]. Fruit weight (FW) and fruit diameter (FD) are typical quantitative traits controlled by polygenes. The QTLs for FW were linked to LG 6 [10], LG 2, 4, 6 [7], LG 2, 4, 5 [42], LG 1, 4, 6, 7 [43] and LG $1,2,3,5,6,7$ [44]. FD QTLs were found in linkage groups 1, 2, 3, and 7 [42]. García-Gómez et al. (2019) reported that in ' $\mathrm{G} \times \mathrm{C}$ ' progeny, the most important QTLs for FW on LG1 and for fruit diameter on LG1 and LG3 [16]. Cao et al. (2016) mapped the association regions for FW on each scaffold by GWAS [45]. We identified QTLs for FW and FD on LG4, 5, and 6. The region of QTL qP-FW4.1 ${ }^{1} \quad$ (Pp04: 15,082,371..16,710,388) was
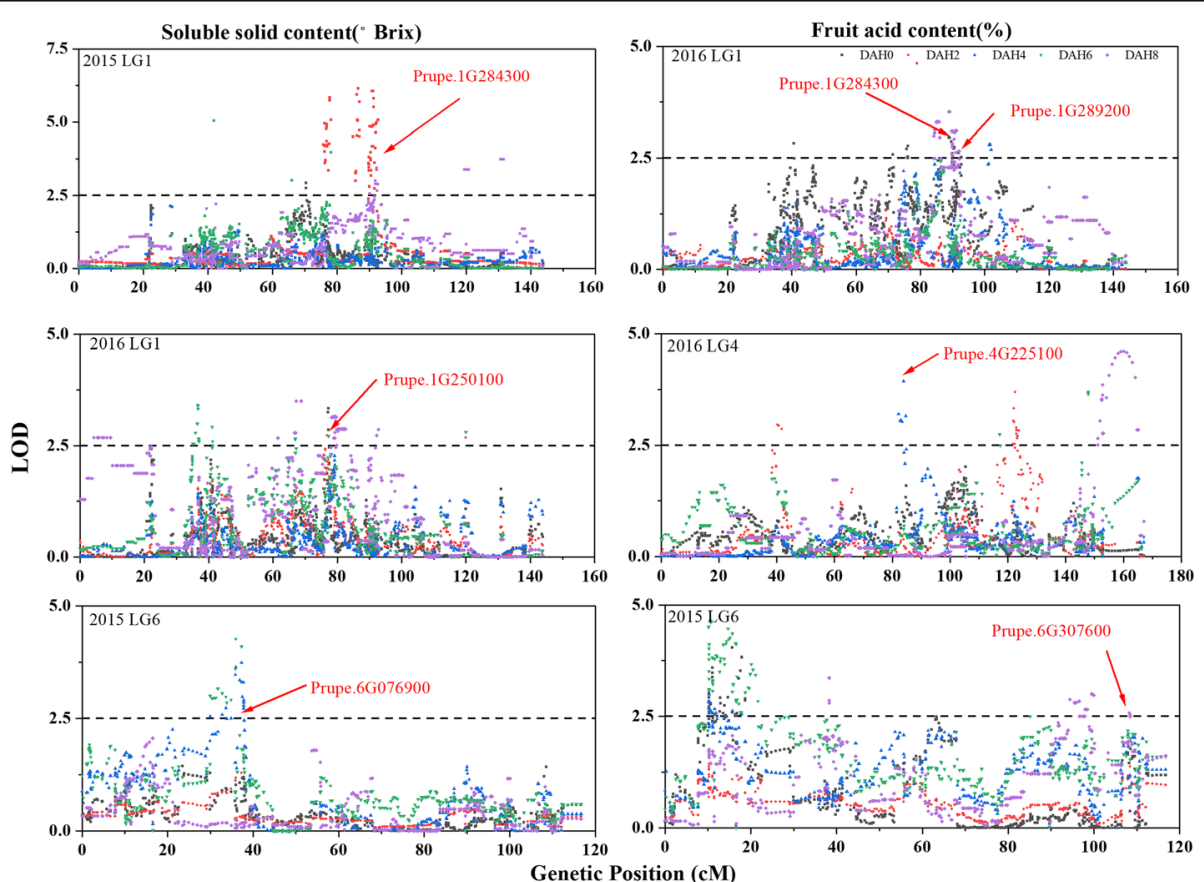

Fig. 3 Dynamic QTLs of soluble solid content (SSC) and fruit acid content (FA) during fruit storage. DAH: day after harvest. The locations of the loci for candidate genes associated with SSC and FA are shown by arrows 

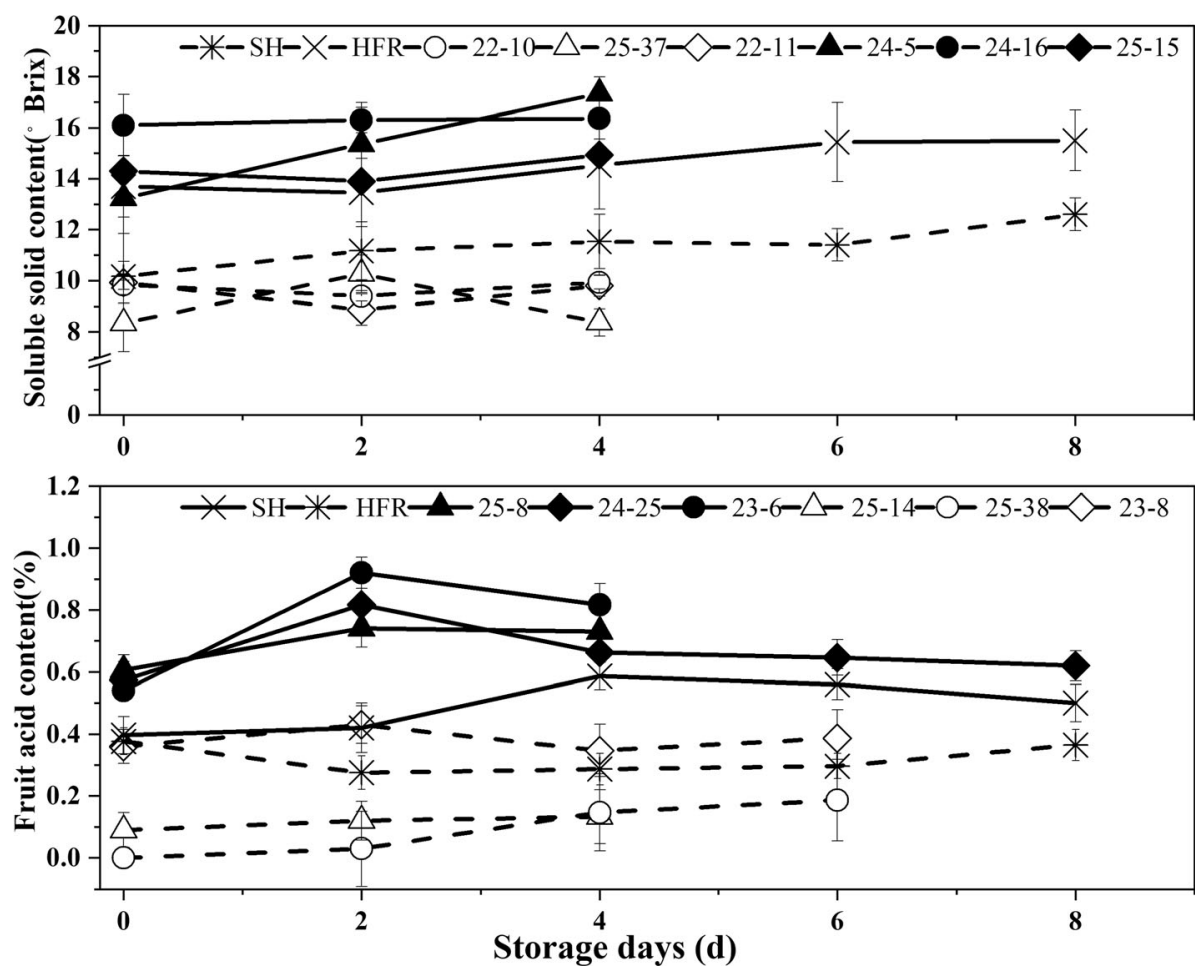

Fig. 4 Change in soluble solid content (SSC) and fruit acid content (FA) in fruits of different genotypes during storage

close to FW-2010-15 (Pp04:17,625,270) [45] and the FW marker SNP_IGA_410336 (Pp04:10,665,019) [44]; QTL qP-FW4.2 (Pp04: 6,316,366.. 6,320,223) was close to FW-2010-12 (Pp04:8,634,341) [45]; qP-FW5.12 (Pp05: 7,318, 892.. 7,816,084) was close to SNP_IGA_574551(Pp05: 6,287, 541) [41], and FW-2010-19 (Pp05: 9432987) [45]; qPFW6.1 ${ }^{2}$ (Pp06: 28,159,465.. 28,159,465) was close to FW2010-21 (Pp06: 20, 400,960) [45]. In addition, our results also showed that most of the QTLs for FW and FD were highly coincident, which may be due to the significant correlation between fruit weight and fruit diameter.

Pigmentation (skin and flesh colour) is an important fruit quality trait in commercial peaches. Verde et al. (2002) detected 2 QTLs for fruit skin colour; one QTL was placed in LG 2, and the other was placed in LG 6 [46]. Eduardo et al. (2011) reported that QTLs for external colour (EC) were located in LG 3, 6, 7 [7]. Frett (et al., 2014) detected four QTLs for skin blush: one major QTL Blush.Pp.ZC-3.1 on LG3 and three minor QTLs on LG 4 and 7 (Blush.Pp.ZC-4.1; Blush.Pp.ZC-4.2; Blush.Pp.ZC-7.1) [47]. The most significant QTLs were localised in LG3 for skin and flesh colour of apricot (García-Gómez et al., 2019) [16]. In our study, QTLs for PSC were identified in LG $1,3,4$, and 6 . The region of QTL qP-PSC3.1 ${ }^{1}$ (Pp03: 10,648,307.. 10,838,614) overlapped with the major QTL for blush, Blush.Pp.ZC-3.1 on LG3 of the $\mathrm{ZC}^{2}$ SNP linkage map, residing on scaffold 3:
$4,821,129 . .13,891,040$. However, the position of QTL qPPSC4.1 ${ }^{1}$ (Pp04: 19,804,890.. 19,842,35) was different from the minor QTLs Blush.Pp.ZC-4.1 (scaffold _ 4: 2,337, 191..3,966,620) and Blush.Pp.ZC-4.2 (scaffold _ 4: 4,306, $550 . .5,226,293$ ) [47]. In addition, in this study, the red colour in flesh (RF) and red colour around pit (RP) were localized in the regions of Pp01: 24,397,147.. 26,479,742 and Pp01: 26,671,055.. 27,176,678 of LG1, respectively. Yamamoto et al. (2005) reported that flesh colour around the stone was mapped in the middle of LG 3 [18]. Cao et al. (2016) found that the SNP associated with flesh colour around the stone was located on scaffold_6: 2,183, 867, scaffold_8: $16,905,885$, scaffold_8: $16,795,565$, scaffold_1: $31,040,363$, and scaffold_1:45,251,328 [45]. These findings suggested that differences in anthocyanin content in peach flesh may be related to multiple genes.

Fruit flavour was important to consumers and thus was an important target for developing peach cultivars. Soluble sugars and acids are important components of fruit flavour. In our study, QTLs for FV were identified on LG $1,3,4$, and 5 . The region of QTL qP-FV1.2 $2^{2}$ (Pp01: 27,653,862.. 28,166,262) was close to the marker ВРPCT020 (Pp01:34,255,110..34,255,594), which was linked to the glucose, sorbitol, total sugar, and soluble solids content [48]. qP-FV4.1 ${ }^{1}$ (Pp04: 15,270,537.. 17, 635,472) overlapped with SSC-2007-14 (Pp04: 17, 057, 020) [45]. The regions of QTLs qP-FV5.1 $1^{1}$ (Pp05: 638, 


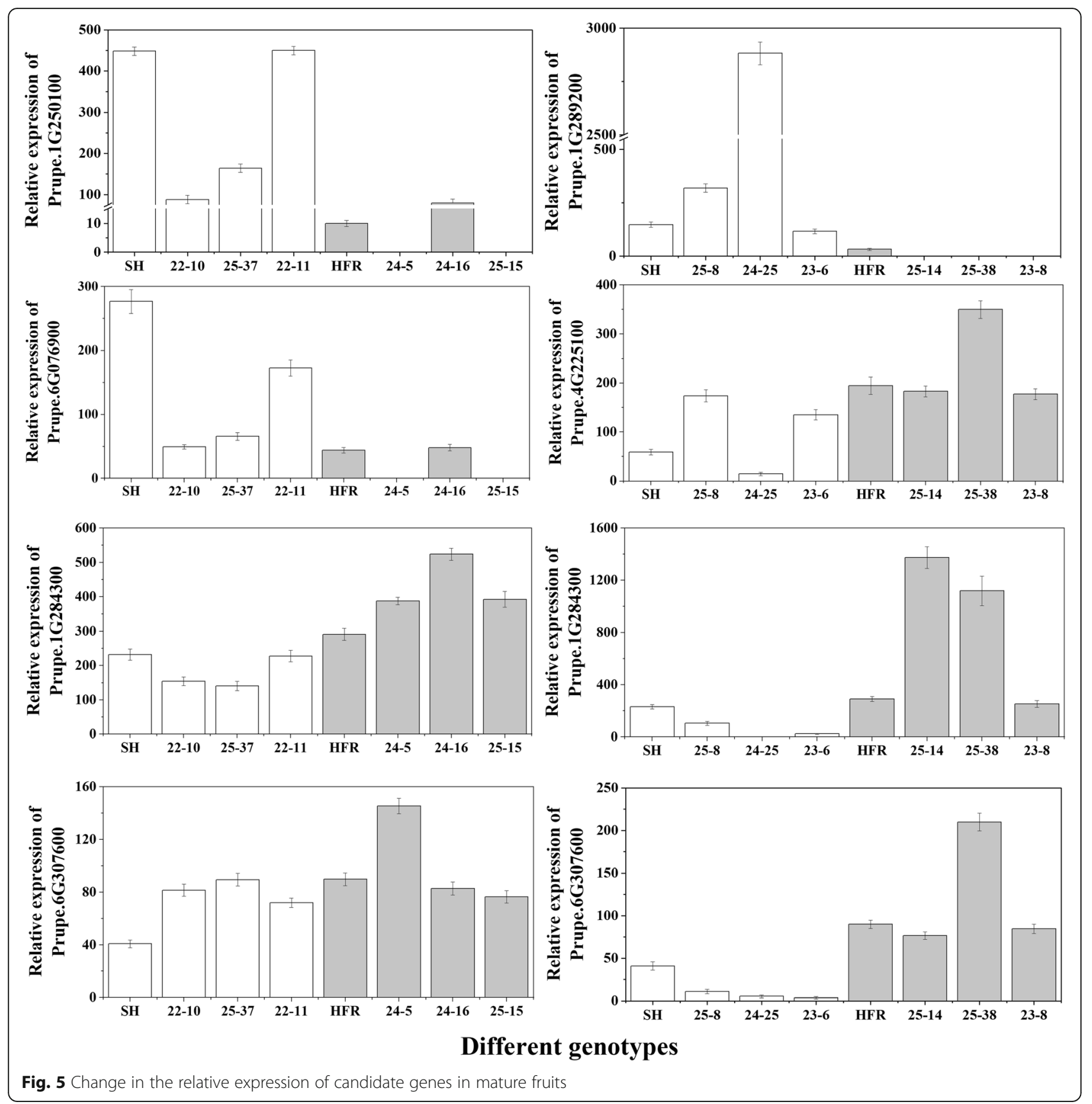

231.. 638,231) and qP-FV5.2 ${ }^{1}$ (Pp05: 1,499,828.. 1499, 831) overlapped with the reported positions for the $D$ locus of acid and non-acid fruit (scaffold_5: 467,067..2, 270,122) [32]. Fresnedo-Ramı'rez et al. (2015) and Boudehri et al. (2009) also found that the locus of G5Flav was associated with the D locus [42, 49]. Furthermore, QTL qP-FV5.1 ${ }^{1}$ (Pp05: 638,231.. 638,231) was also very close to SNP_IGA_544640 (scaffold_5: 629,641), which showed the strongest association with fruit titratable acidity [50].
QTLs for SSC have previously been reported to be linked to peach LG1, 2, 4, 5, 6 [10, 12, 38, 46, 51, 52]. In apricot, García-Gómez et al. (2019) located the most significant QTLs for SSC in LG4 [16]. Our results showed that QTLs for SSC on LG1, 4, 5, and 6. On LG1, the regions of QTLs for SSC (Pp01:13,177,641.. 15,291,518 and Pp01: 24,241,572.. 24,284,070) were very close to the reported positions for SSC-2007-1 (Pp01: 15,691,351) and SSC-2007-4 (Pp01: 24,175,076), respectively [45]. However, in other linkage groups, the QTL positions of 
the SSCs we detected are different from those of other researchers, such as qSSC. V-Ch4-2010 was located in scaffold_4: 17,988,261 [38], QTLSSC-LG5 was located in Pp05:12,106,999..18,240,259 [49], and qSSC.6 was located between the markers ss_629062 $(7,918,349)$ and ss_630302 $(12,571,791)$ on LG6 [42].

For the acid content of fruit, the D major gene controlling the 'non-acid' fruit character was located in LG 5 (scaffold_5: 467,067..2,270,122) [32]. Moreover, QTLs for $\mathrm{pH}$ and titratable acidity were located near the $\mathrm{D}$ gene [10]. We also detected QTL loci for fruit acidity content (FA) on LG5 (Pp05: 629,308.. 677,913 and Pp05: 846,656 . 1,140,022, which was consistent with the D locus. In addition, we also detected QTLs for FA on LG1, 4, and 6. Eduardo et al. (2011) reported that QTLs for FA and fruit $\mathrm{pH}$ were located on LG4 [7]. The region of QTL for FA we detected on LG6 was close to qTA6.1 $(7,550,351 . .8,127,200)$ and qTA6.2(23,319,780.. 26,118, 990) [18].

FDP had a non-normal distribution in progeny according to the Kruskal-Wallis test. QTLs for FDP were detected on LG 1, 2, 3, 4, 5, and 6 [12, 40, 53]. Pirona et al. (2013) located the major QTL qMD4.1 between the markers M12a (Pp04:9,219,594) and ВРPCT023 (Pp04: 14,731,772) [53]. We detected 5 QTLs for FDP, of which two were located on LG 7 and three on LG 4. qPFDF4.3 ${ }^{1}$ (Pp04: 16,420,904) was near qMD4.1.

In summary, although we also identified some new QTLs, most of the mapping results of peach fruit quality traits overlapped or were similar to those of previous studies, which suggests that these genomic regions have important controlling roles on fruit quality traits. These stable QTLs in different genetic populations could be priorities for fine mapping, candidate gene identification and marker-assisted selection (MAS) to improve peach fruit quality.

\section{Candidate genes involved in fruit-related traits in peach}

Candidate genes for fruit-related traits were screened and identified by phenotype-related quantitative trait loci (QTLs). Eduardo et al. (2013) identified candidate genes encoding two putative terpene synthases and one lipoxygenase (Lox), which are involved in the biosynthesis of linalool and p-menth-1-en-9-al, and nonanal, respectively [14]. Pirona et al. (2013) identified NAC (ppa008301m) as a candidate gene controlling maturity date in peach using QTL analysis [53]. PpYUC11 and Prupe.6G150900.1 were identified as candidate genes for controlling the stony hard phenotype in peach $[9,54]$. Nuñez-Lillo et al. (2015) screened five and nine candidate genes for maturity date and mealiness from QTL regions, respectively [3]. Cao et al. (2016; 2019) identified a large number of candidate genes controlling agronomic traits according to a genome-wide association study in peach $[45,55]$. NuñezLillo et al. (2019) identified candidate genes for soluble solid content, maturity date, and mealiness in peach [56]. Carrasco-Valenzuela et al. (2019) identified auxin biosynthetic pathway related genes involved in fruit softening rate by integrating conventional QTL and expression QTL (eQTL) [17]. In apricot, the candidate genes (ppa001122m, ppa000854m and ppb001660m) for the soluble solid were identified which were involved in diglucose and D-mannose binding, and transcription factor MYB10 was found was the best candidate gene for skin colour [16].

In our study, 542 annotated genes were identified for SSC, and these annotated genes included 22 transcription factors and some softening-related cell wall remodelling degradation genes, such as pectin lyase-like superfamily proteins, polygalacturonase, expansin and xyloglucosyl transferase. Etienne et al. (2002) showed the relationship between sugar accumulation and softening processes in fruit development [51]. However, among these candidate genes, none of these genes was annotated as being involved in sugar transport or metabolism. Cao et al. (2016) also showed similar results [45]. However, among the candidate genes for FA, a large number of genes were annotated as being involved in such processes as sugar and acid synthesis, metabolism and transport, such as UDP-glucose 6-dehydrogenase (Prupe.1G284300), glucose-6-phosphate dehydrogenase (Prupe.6G307600), fructose-2,6-bisphosphatase (Prupe.6G053800), fructokinase-like (Prupe.1G289300), sucrose transporter (Prupe.1G271500), sugar transporter protein (Prupe.2G024100), V-ATPases (Prupe.4G225100), and vacuolar proton ATPase (Prupe.6G092300). UDPglucose 6-dehydrogenase participates in the metabolic starch and sucrose metabolism pathways [57]. Glucose-6phosphate dehydrogenase (G6PDH) has been shown to control the non-reversible dehydrogenation of glucose-6phosphate concomitant with the reduction of NADP to NADPH [58]. The qPCR results showed that the expression of Prupe.1G284300 and Prupe.6G307600 in the parents and hybrids was positively correlated with SSC and negatively correlated with FA in the mature stages of fruits. These data suggested that Prupe.1G284300 and Prupe.6G307600 may be involved in both sugar and acid formation of peach fruits. However, further study is warranted to determine the exact function of candidate genes in the formation of SSC and FA traits in peach fruit.

By SLAF-based SNP markers, we constructed the higher density genetic maps, and identified a large number of QTLs and candidate genes for fruit quality traits, especially in terms of fruit flavor and eating quality. Fruit taste perception is not only affected by the SSC and acid 
content. In our study, QTLs for fruit flavour co-located with large amounts of sugar and acidity related traits, and involved in the processes as carbon metabolism, citrate cycle, and fructose and mannose metabolism, and starch and sucrose metabolism. The evaluation of intrinsic quality may provide original data for a comprehensive evaluation of fruit quality as related to its commercial potential [2]. These results are of interest to efforts to better understand the genetic mechanism of intrinsic peach quality, and also can be used to design appropriate breeding strategies to improve the intrinsic quality of commercial peach cultivars, which is the major importance for consumer.

\section{Conclusion}

In this study, we constructed a high-density genetic map in peach based on the SLAF-seq method. This map spanned $1098.79 \mathrm{cM}$ with an average distance of $0.17 \mathrm{cM}$ between adjacent markers, and 90 QTLs for fruit quality related traits were mapped. From the corresponding genomic regions of these QTLs, a large number of candidate genes controlling fruit quality traits were identified. The candidate genes for fruit flavor are focused on the metabolisms and transportion of citrate, fructose, mannose, and sucrose. Candidate genes for eating quality are enriched in the synthesis and metabolisms of starch, sucrose. For SSC, the candidate genes are involved in the metabolisms of fructose, mannose, and fatty acid. For FA, the candidate genes are related to the metabolisms of starch, sucrose, and C5-branched dibasic acid. According to the qPCR, Prupe.1G284300 and Prupe.6G307600 may be involved in sugar and/or acid trait formation of peach fruits. To improve the selection efficiency for peach fruit-quality traits, the corresponding molecular markers can be developed by CAPS and/or dCAPS methods based on the genomic sequences of important candidate genes. In summary, the high density genetic map, QTLs and candidate genes studied here provide useful information for marker-assisted breeding (MAS) of peach fruit-related traits, and these results establish a foundation for the further QTL analysis, mapbased cloning and functional research.

\section{Methods}

\section{Plant material}

An $F_{1}$ peach population of 202 individuals derived from the cross between Prunus persica cv. 'Shahong' (SH) and Prunus persica cv. 'Hongfurong' (HFR) was used. SH was identified in 1999 by the Crop Variety Examination Committee of Shaanxi Province, China. HFR was identified in 2000 by Beijing Crop Variety Examination Committee, China. Our research team introduced $\mathrm{SH}$ and HFR from the National Fruit Tree Germplasm Repository, Zhengzhou Fruit Research Institute, Chinese
Academy of Agricultural Sciences, in China in 2003. SH was a bud mutation of the 'Kurakato Wase' characterised by a early ripening time, peach with medium fruit weight, fruit skin partly coloured, medium sugar content and acidity and clingstone. HFR had been developed from a cross between 'Qiuyu' and 'Xiufeng' characterised by a late ripening time, nectarine with medium fruit weight, fruit skin partly coloured, high sugar content, low acidity and semi-freestone. Two hundred two seedlings of $F_{1}$ offspring were grown in a nursery in 2008 and planted in the next spring on their own roots $(4 \times$ $1.0 \mathrm{~m})$ in a field at the Peach Experimental Demonstration Station of Northwest A\&F University $\left(33^{\circ} 59^{\prime} \mathrm{N}\right.$, $\left.107^{\circ} 39^{\prime} E\right)$, Shaanxi Province, China. Parents and hybrids were grown under natural rainfall conditions with no irrigation, and NPK fertilizer was applied every spring. Pruning was performed yearly, and pests and diseases were controlled by conventional techniques. Hand thinning was carried out before pit hardening to a load of 60-90 fruits per tree. Fifteen fruits per tree were harvested at commercial maturity based on visual colour change and the index of absorbance difference (IAD). Fruits with an IAD between 0.8 and 1.5 were selected [59]. In addition, within the $F_{1}$ progenies, 12 genotypes (22-10, 22-11, 23-6, 23-8, 24-5, 24-16, 24-25, 25-8, $25-14,25-15,25-37$ and 25-38) were selected for qPCR analysis. These genotypes were selected because 24-5, 24-16 and 25-15 had high soluble solid content (SSC), while 22-10, 22-11, and 25-37 had low SSC; $23-$ $6,24-25$ and $25-8$ had high fruit acidity content (FA), while $23-8,25-14$ and $25-38$ had low FA.

DNA extraction, SLAF library construction and sequencing Genomic DNA of parents and progenies was extracted from young leaves using the plant genomics DNA kit (Tiangen, Beijing, China) following the manufacturer's protocol. The concentration and quality of DNA were examined by electrophoresis in 1\% agarose gels and an ND-1000 spectrophotometer (NanoDrop, Wilmington, DE, USA). The SLAF-seq strategy of high-throughput sequencing was used for library construction. Briefly, the reference genome of Prunus persica L. (http://www.ncbi. nlm.nih.gov/genome/388) was used to select restriction enzyme combinations. RsaI and HaeIII (New England Biolabs, NEB, USA) were applied to digest the genomic DNA from each sample. The digested fragments were subjected to the addition of single-nucleotide $\mathrm{A}$ at their 3 '-ends. The ligation of sequencing adapters, PCR amplification, and purification and sequencing of PCR products followed the manufacturer's recommendations, in which the PCR fragments ranging from 264 to $364 \mathrm{bp}$ were purified, and the sequencing was performed using an Illumina HiSeq 2500 system (Illumina Inc., San Diego, CA, USA). 


\section{SLAF-seq data analysis and genotyping}

The identification and genotyping of SLAF markers was carried out according to the method of Zhang et al. [27, 60]. Briefly, after filtering out the low-quality reads (quality score $<20 \mathrm{e}$ ), the remaining reads were sorted to each progeny based on duplex barcode sequences. The SOAP software was used to map the clean reads with terminal $5 \mathrm{bp}$ trimmed onto the peach reference genome [61]. The threshold for definition of a SLAF locus was over 95\% sequence identity, and alleles in each SLAF locus were defined by the minor allele frequency evaluation. Single nucleotide polymorphisms (SNPs) were detected between parents using the software GATK (https://software.broadinstitute.org/gatk/best-practices/ \#variant-disco) and BWA (http://bio-bwa.sourceforge. net/), and SLAFs with > three SNPs were removed. SLAFs with more than four alleles were defined as repetitive SLAFs and discarded.

All polymorphic SLAF loci were genotyped according to the parental and offspring SNP loci. The analysis of the marker code of polymorphic SLAFs was carried out based on the software HighMap with a cross-pollinator population type (a cross between two heterozygous diploid parents), which was composed of five segregation types $(\mathrm{ab} \times \mathrm{cd}$, ef $\times \mathrm{eg}, \mathrm{hk} \times \mathrm{hk}, \mathrm{lm} \times \mathrm{ll}$ and $\mathrm{nn} \times \mathrm{np})$. However, only three segregation types $(\operatorname{lm} \times 1 \mathrm{ll}, \mathrm{nn} \times \mathrm{np}$ and $h k \times h k)$ were genotyped in this paper. To ensure the quality of the genetic map, the valid loci for genetic mapping were filtered using the following rules. First, the lower depth genotype was set as missing, and those with more than 10 missing data points at each locus were eliminated. Second, a chi-square test was performed, and the threshold $P$-value was set to 0.01 . The ' $\mathrm{lm} \times \mathrm{ll}$ ' and ' $\mathrm{nn} \times \mathrm{np}$ ' types had segregation ratios of 1 : 1 , while that of ' $\mathrm{hk} \times$ hk' was 1:2:1. Finally, SNPs with less than $70 \%$ integrity and parental markers that were not homologous for polymorphisms were treated the same way.

\section{Phenotypic data of fruit-related traits}

Phenotypic identification for fruit quality characteristics in peach was performed in 2015 and 2016 according to the method of Frett et al. (2012) and Wang et al. (2005) $[62,63]$. The fruit development period (FDP) was the number of days from full bloom to fruit ripening. Fruit weight (FW) and fruit diameter (FD) were measured as the average of 10 random fruit samples from each tree. The percentage of red skin colour (PSC) was a visual estimation of the surface covered. Red in flesh (RF) and red around pit (RP) were determined separately by visual estimation of the presence of red in flesh and around pit, which was scored as present 1 or absent 0 (Table 1 ). Flesh adherence to pit (AP) was recorded as freestone (flesh and pit completely separate), semi-freestone (flesh partially separates from pit) and clingstone (no separation between flesh and pit) (Table 3). Eating quality $(\mathrm{EQ})$, fruit flavour (FV) and fruit fibre content (FFC) were determined by tasting estimation of nine breeders together with at least ten ripe fruits from each tree. EQ and FV were measured qualitatively on a scale from 1 to 5 , and FFC was measured from 1 to 3 (Table 3).

Fruit samples for measuring soluble solid content (SSC, average brix degrees) and fruit acidity content (FA) were also collected at commercial maturity and were stored at $25 \pm 0.5^{\circ} \mathrm{C}$ with a relative humidity of $75-85 \%$. The SSC and FA of the fruit (at least 5 fruits each time) were measured at intervals of one day during storage until the average firmness was less than $1 \mathrm{~kg} / \mathrm{cm}^{2}$. SSC was measured using a refractometer (ATAGO, model PAL-1), and FA was measured using a fruit acidity meter (Korea, model GMK-835F). The methods of phenotype standardization for 12 fruit quality traits are shown in Table 3.

Table 3 The methods of phenotype standardized for peach fruit quality traits

\begin{tabular}{|c|c|}
\hline Trait & Unit of measure \\
\hline Fruit development period (FDP) & days from full bloom to fruit ripening $(\mathrm{d})$ \\
\hline Fruit weight (FW) & grams \\
\hline Fruit diameter (FD) & the diameter across cheek area $(\mathrm{mm})$ \\
\hline Percentage of red skin color (PSC) & $\%$ \\
\hline Red in flesh (RF) & $0=$ no red overlay; $1=$ red overlay \\
\hline Red around pit (RP) & $0=$ no red; $1=$ red \\
\hline Adherence to pit (AP) & $1=$ freestone; 2 = semi-freestone; $3=$ clingstone \\
\hline Eating quality (EQ) & $1=$ extremely poor $; 2=$ poor; $3=$ fair $; 4=$ good $; 5=$ excellent \\
\hline Fruit flavor (FV) & $1=$ sour $; 2=$ sour-sweet $; 3=$ water sweet $; 4=$ sweet $; 5=$ rich sweet \\
\hline Fruit fiber content (FFC) & $1=$ few $; 2$ = intermediate; 3 = many \\
\hline Soluble solid content (SSC) & $\%$ \\
\hline Fruit acidity content (FA) & $\%$ \\
\hline
\end{tabular}




\section{Linkage map construction and QTL analysis}

HighMap software (http://highmap.biomarker.com.cn/.) was used for linkage map construction [64]. The SLAF markers were mapped to the peach reference genome based on locations and then partitioned into eight linkage groups (LGs). The modified logarithm of odds (MLOD) scores between markers were calculated, and the SLAF markers that scored less than 5.0 were eliminated. The genetic distance in centimorgans $(\mathrm{cM})$ was calculated using Kosambi's mapping function. Quality assessment of the linkage map in terms of collinearity analysis. MapChart 2.3 (https://www.wur.nl/en/show/Mapchart-2.30. $\mathrm{htm})$ was used to make linkage group figures.

MapQTL6.0 software (https://www.kyazma.nl/index. $\mathrm{php} / \mathrm{mc}$.MapQTL/sc.Evaluate/) was used for QTL mapping. The Kruskal-Wallis test was used to detect candidate QTLs $(P$ value $<0.01)$. Additionally, QTLs with LOD scores greater than the threshold at a 0.99 confidence level based on a 1000-permutation test were declared significant. Neighbouring associated loci having the highest LOD scores $(P<0.02)$ were selected as cofactors in the multiple QTL model analysis.

\section{Identification of candidate genes}

Mapping-associated markers were used to identify the homologous regions of QTLs on the physical map. Corresponding genes in QTLs were referred to the peach genome from GDR [65]. The corresponding genes in QTLs for each trait were mapped to the KEGG database (fttp://fttp.genome.jp/pub/kegg/pathway) for KEGG pathway enrichment analyses. KEGG terms with corrected $P$ values $<0.05$ were considered to be significantly enriched.

\section{RNA extraction and gene expression analysis using real- time quantitative PCR (qPCR)}

Total RNA was isolated from the peach flesh using a modified PowerPlant ${ }^{\circ}$ RNA Isolation Kit. RNA quality and integrity were detected by ultraviolet spectrophotometer and agarose gel electrophoresis. The PrimeScript RT Reagent Kit gDNA Eraser (Takara, Beijing, China) was used for converting total RNA to cDNA.

The primer sequences for qPCR were designed by Beacon Designer 8.0 (Table S3). qPCR was carried out with an iQ5 real-time PCR system (BioRad, Plano, TX, USA). The PCR was completed in a $10 \mu \mathrm{l}$ volume containing $1 \mu \mathrm{l}$ cDNA, $1 \mu \mathrm{l}$ of each primer, $2 \mu \mathrm{l} \mathrm{dd}_{2} \mathrm{O}$ and $5 \mu \mathrm{l}$ SYBR Premix Ex Taq II $(2 \times)$ (Takara). The qPCR programme was as follows: $1 \mathrm{~min}$ at $95^{\circ} \mathrm{C}$, followed by 40 cycles of $15 \mathrm{~s}$ at $95^{\circ} \mathrm{C}, 20 \mathrm{~s}$ at $60^{\circ} \mathrm{C}$ and $20 \mathrm{~s}$ at $72^{\circ} \mathrm{C}$. The mixed sample was heated to $95^{\circ} \mathrm{C}$ for $10 \mathrm{~s}$ and cooled to $65^{\circ} \mathrm{C}$ for $15 \mathrm{~s}$. The sample was then heated to $95^{\circ} \mathrm{C}$ at a rate of $0.1^{\circ} \mathrm{C} / \mathrm{s}$ for melting curve analyses. Peach $18 \mathrm{~S}$ ribosomal RNA (18S rRNA) was used as the reference gene. Relative expression levels were analysed using the $2^{-\Delta \Delta \mathrm{Ct}}$ method. Each sample was analysed in triplicate.

\section{Supplementary information}

Supplementary information accompanies this paper at https://doi.org/10 1186/s12870-020-02557-3.

\begin{abstract}
Additional file 1 Figure S1. Genetic map constructed by SNP markers. A black bar indicates an SLAF marker. The $x$-axis represents linkage group number, and the $y$-axis indicates genetic distance (centimorgan as unit).

Additional file $\mathbf{2}$ Figure S2. Collinearity analysis of mapping marker locations on the genetic map and peach genome. The $x$-axis indicates the genetic distance of each peach $L G$, and the $y$-axis represents the physical length of the LG. Markers on the map are plotted as dots.
\end{abstract}

Additional file 3 Figure S3. QTL location of fruit-related traits. Linkage groups, genetic distances (in centimorgans) and marker names are shown, respectively, on the top, left and right of each linkage group. QTLs are drawn by mapchart software with different RGB colours, and different traits are identified by different colours. QTLs are represented by block vertical bars positioned at the right of each linkage group. Thin lines correspond to LOD-2, and black bars correspond to the LOD-1 confidence interval

Additional file 4 Fig. S4 KEGG pathway enrichment analysis for candidate genes of fruit soluble solid content.

Additional file 5 Fig. S5 KEGG pathway enrichment analysis for candidate genes of fruit acidity content.

Additional file 6 Fig. S6 KEGG pathway enrichment analysis for candidate genes of fruit weight.

Additional file 7 Fig. S7 KEGG pathway enrichment analysis for candidate genes of fruit flavour.

Additional file 8 Fig. $\mathbf{8} 8$ KEGG pathway enrichment analysis for candidate genes of fruit eating quality.

Additional file 9 Fig. $\mathbf{9}$ KEGG pathway enrichment analysis for candidate genes of red in flesh.

Additional file $\mathbf{1 0}$ Table S1. Candidate genes related to fruit soluble solid content (SSC) and acid content (FA).

Additional file $\mathbf{1 1}$ Table S2. Candidate genes related to fruit quality traits, including fruit weight, fruit diameter, fruit development period, percentage of red skin colour, fruit eating quality, fruit flavour, red in flesh, red around pit, adherence to pit and fruit fibre content.

Additional file $\mathbf{1 2}$ Table S3. List of primers used in this study.

\section{Abbreviations}

FW: Fruit weight; FD: Fruit diameter; PSC: Percentage of red skin colour; EQ: Eating quality; RF: Red in flesh; RP: Red around pit; AP: Adherence to Pit; FDP: Fruit development period; FV: Fruit flavour; FFC: Fruit fibre content; SSC: Soluble solid content; FA: Fruit acid content; IM: Interval mapping; MQM: Multiple QTL models; CM: Centimorgan; LG: Linkage group; chr: Chromosome; LOD: Logarithm of odds; QTL: Quantitative trait loci; AFLP: Amplified Fragment Length Polymorphism; RAPD: Random Amplified Polymorphism DNA; RFLP: Restriction Fragment Length Polymorphism; SSR: Simple Sequence Repeat; SLAF-seq: Specific locus amplified fragment sequencing; SNP: Single nucleotide polymorphism

\section{Acknowledgements}

We are grateful to Fu-rui Li and Lei Zhao for their assistance in sample collection.

\section{Authors' contributions}

PS carried out all experiments and data analysis. CPZ, MYH, LBX, JJM and DZ analyzed the results and modified the manuscript. PS, ZX, SYZ, XJW, XFM and $J C Z$ performed phenotyping of 14 fruit-related traits. The authors read and approved the final manuscript. 


\section{Funding}

This work was supported by grants from National Key R\&D Program of China (Grant No. 2019YFD1000200) and Key Research \& Development program of Shaanxi Province, China (Grant No. 2018NY-048). The funder had no roles in the design of the study and collection, analysis, and interpretation of data and in the writing the manuscript.

\section{Availability of data and materials}

The datasets of raw sequence data during the current study are available in the Sequence Read Archive (SRA) under accession number PRJNA612130 [https://www.ncbi.nIm.nih.gov/bioproject/PRJNA612130]. All other data generated or analysed during this study are included in this published article and its supplementary information files.

\section{Ethics approval and consent to participate}

Not applicable.

\section{Consent for publication}

Not applicable.

\section{Competing interests}

The authors declare that they have no conflict of interest.

\section{Received: 19 March 2020 Accepted: 19 July 2020}

Published online: 23 September 2020

\section{References}

1. Verde I, Abbott AG, Scalabrin S, Jung S, Shu S, Marroni F, et al. The highquality draft genome of peach (Prunus persica) identifies unique patterns of genetic diversity, domestication and genome evolution. Nat Genet. 2013; 45(5):487-94.

2. Infante RM-GP, Predieri S. Quality oriented fruit breeding: peach (Prunus persica (L.) Batsch). J Food Agriculture Environ. 2008;6(2):342-56.

3. Nunez-Lillo G, Cifuentes-Esquivel A, Troggio M, Micheletti D, Infante R, Campos-Vargas $R$, et al. Meneses $C$ : identification of candidate genes associated with mealiness and maturity date in peach [Prunus persica (L.) Batsch] using QTL analysis and deep sequencing. Tree Genet Genomes. 2015;11(4):86

4. Chaparro JX, Werner DJ, Omalley D, Sederoff RR. Targeted mapping and linkage analysis of morphological Isozyme, and Rapd markers in peach. Theor Appl Genet. 1994;87(7):805-15.

5. Sosinski B, Sosseyalaoui K, Rajapakse S, Glassmoyer K, Ballard RE, Abbott AG, et al. Use of AFLP and RFLP markers to create a combined linkage map in peach [Prunus Persica (L.) Batsch] for use in marker assisted selection. Acta Hortic. 1998:465(465):61-8.

6. Dirlewanger E, Cosson P, Boudehri K, Renaud C, Capdeville G, Tauzin Y, et al. Development of a second-generation genetic linkage map for peach [Prunus persica (L.) Batsch] and characterization of morphological traits affecting flower and fruit. Tree Genet Genomes. 2006;3(1):1-13.

7. Eduardo I, Pacheco I, Chietera G, Bassi D, Pozzi C, Vecchietti A, et al. QTL analysis of fruit quality traits in two peach intraspecific populations and importance of maturity date pleiotropic effect. Tree Genet Genomes. 2011; 7(2):323-35.

8. Martinez-Garcia PJ, Parfitt DE, Ogundiwin EA, Fass J, Chan HM, Ahmad R, et al. High density SNP mapping and QTL analysis for fruit quality characteristics in peach (Prunus persica L.). Tree Genet Genomes. 2013;9(1): 19-36.

9. Guo SL, labal S, Ma RJ, Song J, Yu ML, Gao ZH. High-density genetic map construction and quantitative trait loci analysis of the stony hard phenotype in peach based on restriction-site associated DNA sequencing. BMC Genomics. 2018;19(1):612.

10. Dirlewanger E. Mapping QTLs controlling fruit quality in peach (Prunus persica (L.) Batsch). Theoretical and Applied Genetics 1999; 98(1):18-31.

11. Fresnedo-Ramirez J, Frett TJ, Sandefur PJ, Salgado-Rojas A, Clark JR, Gasic K, et al. QTL mapping and breeding value estimation through pedigree-based analysis of fruit size and weight in four diverse peach breeding programs. Tree Genet Genomes. 2016;12(2):25

12. Hernandez Mora JR, Micheletti D, Bink M, Van de Weg E, Cantin C, Nazzicari $\mathrm{N}$, et al. Integrated QTL detection for key breeding traits in multiple peach progenies. BMC Genomics. 2017;18(1):404.
13. Sanchez G, Martinez J, Romeu J, Garcia J, Monforte AJ, Badenes ML, et al. The peach volatilome modularity is reflected at the genetic and environmental response levels in a QTL mapping population. BMC Plant Biol. 2014;14(1):137.

14. Eduardo I, Chietera G, Pirona R, Pacheco I, Troggio M, Banchi E, et al. Genetic dissection of aroma volatile compounds from the essential oil of peach fruit: QTL analysis and identification of candidate genes using dense SNP maps. Tree Genet Genomes. 2013;9(1):189-204.

15. Bielenberg DG, Rauh B, Fan SH, Gasic K, Abbott AG, Reighard GL, et al. Genotyping by sequencing for SNP-based linkage map construction and QTL analysis of chilling requirement and bloom date in peach [Prunus persica (L.) Batsch]. PLoS One. 2015;10(10):e0139406.

16. García-Gómez BE, Salazar JA, Dondini L, Martínez-Gómez P, Ruiz D. Identification of QTLs linked to fruit quality traits in apricot (Prunus armeniaca L.) and biological validation through gene expression analysis using qPCR. Mol Breed. 2019;39(2):28.

17. Carrasco-Valenzuela T, Muñoz-Espinoza C, Riveros A, Pedreschi R, Arús P, Campos-Vargas R, et al. Expression QTL (eQTLs) analyses reveal candidate genes associated with fruit flesh softening rate in peach [Prunus persica $(\mathrm{L}$.) Batsch]. Front Plant Sci. 2019;10:1581

18. Yamamoto T, Yamaguchi M, Hayashi T. An integrated genetic linkage map of peach by SSR, STS, AFLP and RAPD. J Jpn Soc Hortic Sci. 2005;74(3):204-13.

19. Lu ZX, Sosinski B, Reighard GL, Baird WV, Abbott AG. Construction of a genetic linkage map and identification of AFLP markers for resistance to root-knot nematodes in peach rootstocks. Genome. 1998:41(2):199-207.

20. Tataranni G, Spada A, Pozzi C, Bassi D. AFLP-based bulk segregant analysis for tagging the slow-ripening trait in peach [Prunus persica (L.) Batsch]. J Hortic Sci Biotech. 2010;85(1):78-82.

21. Eldredge L, Ballard R, Baird W, Abbott A, Morgens P, Callahan A, et al. Application of RFLP analysis to genetic linkage mapping in peaches. Hortscience. 1992;27(2):160-3.

22. Chen $\mathrm{H}$, Xie W, He H, Yu H, Chen W, Li J, et al. A high-density SNP genotyping array for rice biology and molecular breeding. Mol Plant. 2014; 7(3):541-53.

23. Wu J, Li LT, Li M, Khan MA, Li XG, Chen $H$, et al. High-density genetic linkage map construction and identification of fruit-related QTLs in pear using SNP and SSR markers. J Exp Bot. 2014;65(20):5771-81.

24. Zhang J, Zhang QX, Cheng TR, Yang WR, Pan HT, Zhong JJ, et al. Highdensity genetic map construction and identification of a locus controlling weeping trait in an ornamental woody plant (Prunus mume Sieb. Et Zucc). DNA Res. 2015;22(3):183-91.

25. Zhang YX, Wang LH, Xin HG, Li DH, Ma CX, Ding $X$, et al. Construction of a high-density genetic map for sesame based on large scale marker development by specific length amplified fragment (SLAF) sequencing. BMC Plant Biol. 2013;13:141.

26. Zhang Z, Shang H, Shi Y, Huang L, Li J, Ge Q, et al. Construction of a highdensity genetic map by specific locus amplified fragment sequencing (SLAF-seq) and its application to quantitative trait loci (QTL) analysis for boll weight in upland cotton (Gossypium hirsutum.). BMC Plant Biol. 2016:16:79.

27. Zhang J, Zhang Q, Cheng T, Yang W, Pan H, Zhong J, et al. High-density genetic map construction and identification of a locus controlling weeping trait in an ornamental woody plant (Prunus mume Sieb. Et Zucc). DNA Res. 2015;22(3):183-91.

28. He YX, Yuan WJ, Dong MF, Han YJ, Shang FD. The first genetic map in sweet Osmanthus (Osmanthus fragrans Lour.) using specific locus amplified fragment sequencing. Front Plant Sci. 2017;8:1621.

29. Shang JL, Li N, Li NN, Xu YY, Ma SW, Wang JM. Construction of a highdensity genetic map for watermelon (Citrullus lanatus L.) based on largescale SNP discovery by specific length amplified fragment sequencing (SLAF-seq). Sci Hortic-Amsterdam. 2016;203:38-46.

30. Li X, Singh J, Qin M, Li S, Zhang X, Zhang M, et al. Development of an integrated 200K SNP genotyping array and application for genetic mapping genome assembly improvement and genome wide association studies in pear (Pyrus). Plant Biotechnol J. 2019;17(8):1582-94.

31. Verde I, Bassil N, Scalabrin S, Gilmore B, Lawley CT, Gasic K, et al. Development and evaluation of a 9K SNP Array for peach by internationally coordinated SNP detection and validation in breeding Germplasm. PLOS One. 2012:7(4):e35668.

32. Dirlewanger E, Pronier V, Parvery C, Rothan C, Guye A, Monet R. Genetic linkage map of peach [Prunus persica (L.) Batsch] using morphological and molecular markers. Theor Appl Genet. 1998;97(5-6):888-95. 
33. Blenda AV, Verde I, Georgi LL, Reighard GL, Forrest SD, Munoz-Torres M, et al. Construction of a genetic linkage map and identification of molecular markers in peach rootstocks for response to peach tree short life syndrome. Tree Genet Genomes. 2007:3(4):341-50.

34. Ogundiwin EA, Peace CP, Gradziel TM, Parfitt DE, Bliss FA, Crisosto CH. A fruit quality gene map of Prunus. BMC Genomics. 2009;10(1):587.

35. Li TL, Zhang J, Huang L, Han F, Yan S, Wang L, et al. Construction of a highdensity genetic map based on large-scale markers developed by specific length amplified fragment sequencing (SLAF-seq) and its application to QTL analysis for isoflavone content in Glycine max. BMC genomics. 2014;15(1): 1086.

36. Wang FR, Zhang JX, Chen Y, Zhang CY, Gong JW, Song ZQ, et al. Identification of candidate genes for key fibre-related QTLs and derivation of favourable alleles in Gossypium hirsutum recombinant inbred lines with G. barbadense introgressions. Plant Biotechnol J. 2020;18(3):707-20.

37. Lambert P, Campoy JA, Mauroux J-B, Rossini L, Aranzana MJ, Patocchi EDA, et al. Identifying SNP markers tightly associated with six major genes in peach [Prunus persica (L.) Batsch] using a high-density SNP array with an objective of marker-assisted selection (MAS). Tree Genet Genomes. 2016; 4(4):647-661.

38. Zeballos JL, Abidi W, Gimenez R, Monforte AJ, Moreno MA, Gogorcena Y. Mapping QTLs associated with fruit quality traits in peach [Prunus persica (L.) Batsch] using SNP maps. Tree Genet Genomes. 2016;12(3):1-17.

39. Giménez R, Monforte A J, Moreno M A, et al. QTL analysis of fruit quality traits in peach [Prunus persica (L.) Batsch] using dense SNP maps. VIII International Peach Symposium 1084. 2013;703-10.

40. Dirlewanger E, Cardinet G, Boudehri K, Renaud C, Monllor S, Illa E, et al. Detection of QTLs controlling major fruit quality components in peach within the European project ISAFRUIT. Acta Hortic. 2009;814:533-38.

41. Quilot B, Kervella J, Genard M, Lescourret F. Analysing the genetic control of peach fruit quality through an ecophysiological model combined with a QTL approach. J Exp Bot. 2005;56(422):3083-92.

42. Fresnedo-Ramirez J, Bink MCAM, van de Weg E, Famula TR, Crisosto $\mathrm{CH}$, Frett TJ, et al. QTL mapping of pomological traits in peach and related species breeding germplasm. Mol Breed. 2015;35(8):166.

43. Desnoues E, Baldazzi V, Genard M, Mauroux JB, Lambert P. Confolent C, et al. Dynamic QTLs for sugars and enzyme activities provide an overview of genetic control of sugar metabolism during peach fruit development. J Exp Bot. 2016;67(11):3419-31.

44. da Silva LC, Bassi D, Bianco L, Pacheco I, Pirona R, Rossini L. Genetic dissection of fruit weight and size in an F2 peach (Prunus persica (L.) Batsch) progeny. Mol Breed. 2015;35(2):71.

45. Cao K, Zhou ZK, Wang Q, Guo J, Zhao P, Zhu GR, et al. Genome-wide association study of 12 agronomic traits in peach. Nat Commun. 2016;7(1):1-10.

46. Verde I, Quarta R, Cedrola C, et al. QTL analysis of agronomic traits in a BC1 peach population. Acta Hortic. 2002;592:291-7.

47. Frett TJ, Reighard GL, Okie WR, Gasic K. Mapping quantitative trait loci associated with blush in peach [Prunus persica (L.) Batsch]. Tree Genet Genomes. 2014:10(2):367-81.

48. Shen ZJ, Ma RJ, Yu ML, Cai ZX, Xu JL. Mapping QTLs controlling flavor quality of peach using a cross population between nectarine and flat nectarine. Acta Horticulturae Sinica. 2010;37(11):1735-44.

49. Boudehri K, Bendahmane A, Cardinet G, Troadec C, Moing A, Dirlewanger E. Phenotypic and fine genetic characterization of the D locus controlling fruit acidity in peach. BMC Plant Biol. 2009:9(1):59.

50. Micheletti D, Dettori MT, Micali S, Aramini V, Pacheco I, Linge CD, et al. Whole-genome analysis of diversity and SNP-major Gene Association in peach Germplasm. PLoS One. 2015;10(9):e0136803.

51. Etienne C, Rothan C, Moing A, Plomion C, Bodenes C, Svanella-Dumas L, et al. Candidate genes and QTLs for sugar and organic acid content in peach [Prunus persica (L.) Batsch]. Theor Appl Genet. 2002;105(1):145-59.

52. Quilot B, Wu BH, Kervella J, Genard M, Foulongne M, Moreau K. QTL analysis of quality traits in an advanced backcross between Prunus persica cultivars and the wild relative species P-davidiana. Theor Appl Genet. 2004;109(4): 884-97.

53. Pirona R, Eduardo I, Pacheco I, Linge CD, Miculan M, Verde I, et al. Fine mapping and identification of a candidate gene for a major locus controlling maturity date in peach. BMC Plant Biol. 2013:13(1):166

54. Pan L, Zeng W, Niu L, Lu Z, Liu H, Cui G, et al. PpYUC11, a strong candidate gene for the stony hard phenotype in peach (Prunus persica L. Batsch), participates in IAA biosynthesis during fruit ripening. J Exp Bot. 2015;66(22): $7031-44$.

55. Cao K, Li Y, Deng CH, Gardiner SE, Zhu G, Fang W, et al. Comparative population genomics identified genomic regions and candidate genes associated with fruit domestication traits in peach. Plant Biotechnol J. 2019; 17(10):1954-70

56. Nunez-Lillo G, Balladares C, Pavez C, Urra C, Sanhueza D, Vendramin E, et al. High-density genetic map and QTL analysis of soluble solid content, maturity date, and mealiness in peach using genotyping by sequencing. Sci Hortic-Amsterdam. 2019;257:108734.

57. Xue GP, Mclntyre $C L$, Jenkins $C L$, Glassop D, van Herwaarden AF, Shorter R. Molecular dissection of variation in carbohydrate metabolism related to water-soluble carbohydrate accumulation in stems of wheat. Plant Physiol. 2008;146(2):441-54.

58. Kong WF, Chen JY, Hou ZX, Wen PF, Zhan JC, Pan QH, et al. Activity and subcellular localization of glucose-6-phosphate dehydrogenase in peach fruits. J Plant Physiol. 2007;164(7):934-44.

59. Lurie S, Friedman H, Weksler A, Dagar A, Eccher Zerbini P. Maturity assessment at harvest and prediction of softening in an early and late season melting peach. Postharvest Biol Tec. 2013;76:10-6.

60. Sun X, Liu D, Zhang X, Li W, Liu H, Hong W, et al. SLAF-seq: an efficient method of large-scale de novo SNP discovery and genotyping using highthroughput sequencing. PLoS One. 2013;8(3):e58700.

61. Li RQ, Li YR, Kristiansen K, Wang J. SOAP: short oligonucleotide alignment program. Bioinformatics. 2008; 24(5):713-14.

62. Frett TJ, Gasic K, Clark JR, Byrne D, Gradziel T, Crisosto C. Standardized phenotyping for fruit quality in peach (Prunus persica (L.) Batsch). J Am Pomol Soc. 2012;66(4):214

63. Wang L, Zhu G, Fang WC, Ma R, Yu M, Jiang Q, et al. Descripters and data standard for peach. Beijing: China Agriculture Press; 2005.

64. Liu D, Ma C, Hong W, Huang L, Liu M, Liu H, et al. Construction and analysis of high-density linkage map using high-throughput sequencing data. PLoS One. 2014:9(6):e98855.

65. Verde I, Jenkins J, Dondini L, Micali S, Pagliarani G, Vendramin E, et al. The peach v2.0 release: high-resolution linkage mapping and deep resequencing improve chromosome-scale assembly and contiguity. BMC Genomics. 2017:18(1):225.

\section{Publisher's Note}

Springer Nature remains neutral with regard to jurisdictional claims in published maps and institutional affiliations.

Ready to submit your research? Choose BMC and benefit from:

- fast, convenient online submission

- thorough peer review by experienced researchers in your field

- rapid publication on acceptance

- support for research data, including large and complex data types

- gold Open Access which fosters wider collaboration and increased citations

- maximum visibility for your research: over $100 \mathrm{M}$ website views per year

At BMC, research is always in progress.

Learn more biomedcentral.com/submissions 